\title{
ANALYSIS OF COMPLEXITY AND TRANSACTION COSTS FOR CO- OPERATING NETWORKS
}

\author{
Nadeem Akhtar \\ University of Surrey \\ Guildford, UK
}

\author{
Jan Markendahl \\ Wireless@KTH \\ Stockholm, Sweden \\ Klaus Moessner \\ University of Surrey \\ Guildford, UK
}

\section{ABSTRACT}

In this paper we investigate the impact that introduction of new Ambient Networks (AN) functionality will have on usage of system resources and on connection delay. The signalling load for multiple attachment and negotiation procedures is assessed by modelling signalling sequences for a WLAN system enabled with AN technology. The load is computed for varying numbers of users and for users with different levels of "willingness to evaluate and negotiate offers". The results show that the most important parameter is the number of attachment attempts per time unit, which is an indicator of user activity level. In the investigated scenarios, the relative load of signalling is $0.1-1.0 \%$ of the transferred user data. The delay depends on the current load situation of the network.

\section{INTRODUCTION}

The scenarios for next generation communication networks are characterised by four main features. First, there will be a multitude of networks with a large number of access technologies underneath. Second, inter-networking between networks will go beyond what we have today in the form of data forwarding. Interworking between landline and cellular networks is already happening and there is a growing impetus towards Fixed-Mobile Convergence. Third, traditional business roles will disappear and provision of services will be democratised, instead of the current situation where the number of providers is relatively small. In other words, there will be more players besides big operators and providers, as we can already see from the emergence of municipal WLANs, community-based networks such as FON [1] and the growing list of sites that host multimedia content generated by end users. Finally, the relationship between end users and service providers will be much more flexible and dynamic unlike the current subscription-based models. In a highly competitive market, end users would not only like to make the most of what is available but at the same time, choose the best of what is available. Note that the term 'service provider' is used here (and the rest of the paper) in a broad sense to include wired/wireless Internet Service Providers, Mobile Network Operators (MNO), content providers etc.

Based on the future networking vision outline above, it can be inferred that the dominant themes are growing heterogeneity, greater choice of service providers and increased levels of interworking between networks. Therefore, a technology-agnostic framework for dynamic and flexible co-operation is required. The IST Ambient Networks project [2] has proposed the concept of Network Composition for this very purpose. From the point of composition, cooperation includes both business and technical aspects and each such relation between two networks is described by a
Composition Agreement (CA) which is negotiated and realized by them. Co-operation here is meant to encompass any form of interworking between two networks to achieve specific communication goal (s). The simplest case is cooperation between an end user and a network operator to enable the former to make a voice call or access the global Internet. Other examples include co-operation between MNOs for roaming, interworking between access and transit networks to provide QoS guarantees for traffic to/from the access network traversing the transit network.

The composition framework is supported by a set of "tools" to establish the pre-requisites for CA negotiation [3][4][5]. Providers can advertise service offers to end-users. The user devices have functionality for discovery and evaluation of offers and decision making logic for selection of service provider. Prior to $\mathrm{CA}$ negotiation and realization, the two composing networks have to establish a secure communication channel between them. This is done with the help of Ambient Network Attachment Protocol (ANAP) [5].

The composition process is independent of underlying technologies. Furthermore, it is generic and scalable in the sense that it can be used between networks of different types and sizes. Last but not least, it is extensible and hence, it is possible to update a CA that has already been realized. All these properties imply that composition is the ideal solution to enable the dynamic and flexible co-operation between networks, as envisaged in future scenarios. However, it is extremely important to prove the commercial and technical viability of composition. In particular, the complexity of the process and the associated "transaction" costs must be analysed to gain a deeper understanding of the impact of composition various deployment scenarios. To illustrate the feasibility of the composition concept, a major issue is to demonstrate that the added functionality and processes do not introduce "too much" overhead, signaling or delay.

In this paper, we consider the different costs associated with composition and their relative importance for some of the interworking scenarios mentioned previously. The focus is on the amount of signalling used during various phases of composition. Furthermore, we also discuss a methodology that can be used for analyzing the cost of network cooperation. This approach is then exemplified via a combination of analysis and simulation with the help of scenarios with many users and a large number of service providers.

The rest of this paper is organised as follows. In Section II, we discuss the cost and complexity of network co-operation, methodologies for analyzing them are described in Section III. Some examples from related studies performed in the Ambient Networks project are provided in Section IV. Finally, we present a summary of the main findings and the main conclusions in Section V. 


\section{COST AND COMPLEXITY OF INTER-NETWORK CO- OPERATION USING COMPOSITION}

The concept of network composition and its use as an enabler for dynamic and flexible co-operation between networks was briefly discussed in the previous section. It has been designed to have all the desirable properties such as flexibility, scalability, extensibility, technology-independence etc. However, it is also important to make sure that the new functionality is not "too complex" and does not introduce "too large" transaction costs for co-operating networks. In the following, we discuss the main factors that contribute to costs associated with the functionality required to implement composition.

The composition process comprises a number of phases: media sense, network advertisement and discovery, network attachment, CA negotiation and CA realization. Media sensing is essentially a legacy mechanism, hence it is not considered here. The different steps in composition are illustrated by the sequence of actions in Figure 1. A number of operator networks (Ambient Networks, AN 1 - 3) advertise their offers which are detected and evaluated by the "user AN", followed by selection, network attachment, CA negotiation and realisation. The user AN is shown to initiate an application session with another host via AN1 after composition..

The complexity of Network Advertising and Discovery (NAD) depends on a number of factors: nature of NAD process (active/passive), number of Information Elements (IE) and their size, type of information (secure/non-secure), transmission medium (L2/L3), transmission mode (unicast, multicast, broadcast). IEs can be inserted into beacons used for media sensing. Dedicated L2/L3 messages can also be used for this purpose, prior to composition. IEs can be piggybacked onto ANAP messages. Finally, the initial CA proposal itself can be seen as a form of advertising. The implication here is that the NAD phase is not clearly demarcated as advertising can take place at different points of time during the composition process, depending on the nature and amount of information to be advertised. Therefore, the cost of advertising will also vary. The worst case is where all IEs are put into beacons which are broadcast periodically. In this case, it is fairly easy to compute the cost in terms of number of bits sent as well as energy used for transmission/reception per bit. However, in practice, more sophisticated approaches will be used where IEs are distributed in many messages, with different levels of security, during the phases prior to CA negotiation and hence advertising costs must be taken into account while computing the signalling costs for these phases.

The attachment procedure consists of a 4-way handshake. During this phase, security credentials, such as cryptographic identifiers, are exchanged between the two networks. The protocols messages are flexible and can be used to carry advertising payloads. The signalling cost is more or less fixed, except when advertising information is inserted into the ANAP messages.

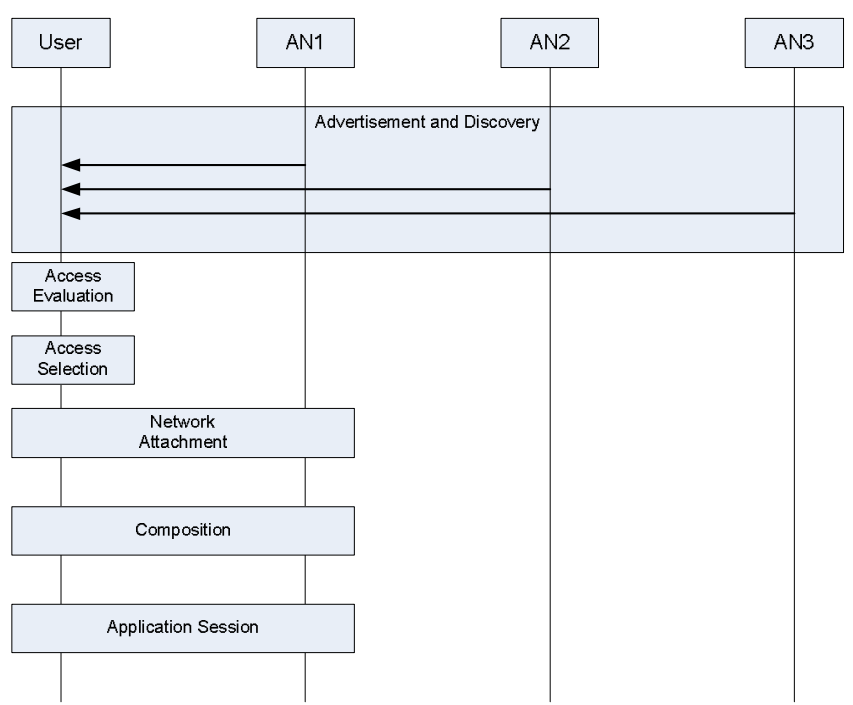

Figure 1: Steps in the composition process

CA negotiation is a critical part of the composition process as the framework for co-operation between the two networks is established during this phase. Negotiations take place in accordance with many different strategies, determined by policies, preferences and the nature of co-operation to be realized. In the simplest case, one of the networks proposes a pre-defined CA to its peer and the choice is "take-it-or-leaveit". Clearly, this is the quickest and least cost-intensive option but at the same time it is highly restrictive. The worst case is where the negotiation process starts with a "blank" CA which then becomes progressively built-up until an agreement is reached between the two parties on all the resources and services under negotiation. This is the most flexible approach but it involves complex signalling and computational intensive interactions. In practice, most negotiations will kick-off with a pre-defined CA template, which is partially filled, leaving room for negotiation over a limited set of aspects, such as Service Level Specifications etc. Besides the complexity associated with template selection and determining what to insert in negotiable fields (if any), there's a signalling cost too. This will depend on the length of negotiations (in terms of number of message round-trips) as well as the size of the CA being exchanged between the two networks.

CA realization is the final step in establishing co-operation and the process involves a 3-way handshake between the two parties for synchronization purposes. The signalling cost is fixed in this case. However, the complexity of realizing a CA depends on the nature of the CA. For instance, it is much simpler to realize a CA for setting up a best-effort VoIP call compared to one which entails provisioning of VPN tunnels between two end-sites. Once realized, a CA can be updated via re-negotiation. This maybe as simple as extending the $\mathrm{CA}$ lifetime but more complex negotiations cannot be ruled out. However, in general, the complexity is somewhat reduced compared to negotiation from scratch. The cost, once again, depends on the length of negotiations and size of CA. Decomposition happens when the lifetime of a CA expires. As this process is more or less the reversal of CA realization, the same considerations apply here. 
So far, the discussion has considered complexity of composition from a more or less "technical" perspective, in terms of signalling and computational cost. Another way to look at is in terms of "transaction costs" which are defined as "costs other than the money that are incurred in trading goods or services" [7].

There are different types of transaction costs e.g. search and information costs, bargaining and decision costs, policing and enforcement costs. Transaction costs can be divided into two categories:

1. Long term (session independent) costs needed to enable the connection including

$>$ Effort and time to establish or enable the agreement or business relation

> Resource (cost) to enable and maintain this type of relation.

2. Short term costs that may occur for every session

D User actions needed - time, delay

$>$ Waiting for response - time, delay

$>$ Resources consumed - system capacity

The consumption of resources includes both signaling between user and providers (B2C signaling) and signaling between providers (B2B signaling) e.g. to check roaming agreements or user identity. Note that composition is assumed to be between end users and a network operator, but this can be generalised to scenarios involving two networks. It must also be pointed out that in the Ambient Networks architecture, "network" is the fundamental building block and end user terminals are also treated as Ambient Networks.

\section{Analysis Methodology}

The key aspects of the modelling scenario are as follows:

- A number of access points (APs) or networks where each AP advertises its services.

- Composition capabilities in APs and user devices

- A number of end users wanting network access that evaluate offers and negotiate conditions

- A number of user strategies for negotiation

To investigate and determine the complexity in terms of signalling load we consider different user strategies where the "user AN" is negotiating with one or more "provider ANs" (see Figure 2 where user AN is represented by boxes labeled "user and terminal"). The most complex case is analyzed in order to provide a sanity check ensuring that Ambient Networks concepts will be feasible also in extreme situations.

The user AN may want to evaluate offers from multiple networks before signing an agreement with one of them. This scenario can have many sub-cases. First, the user AN listens to offers from potential service providers, selects one and proceeds with attachment, negotiation and possibly realisation. Second, the user AN attaches with multiple providers simultaneously, receives CA proposals from them but proceeds with negotiation with only one. Third, it negotiates with several providers in parallel until it reaches an agreement with one of them that fulfils its technical and business requirements. These composition strategies will have significant bearing on the complexity as well as cost.

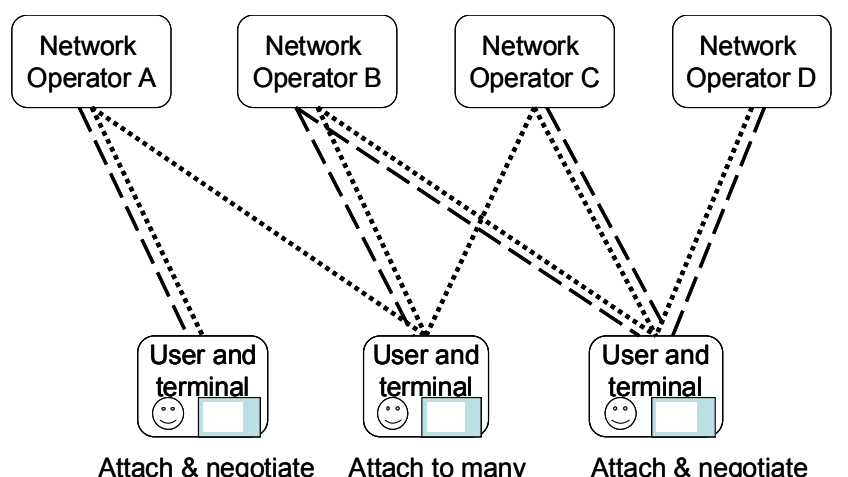

Attach \& negotiate with one network

Attach to many and negotiate with one network

with many networks

\section{Attachment to network}

Negotiation of $\mathrm{CA}-\mathrm{-}-\mathrm{\cdots}-\mathrm{\cdots}$

Figure 2: Complexity cases for the signaling load analysis

In order to estimate the amount of signalling data needed for composition, the process was analysed using a WLAN system. The different parts in the composition process were analyzed at a message level including network attachment, signalling session establishment, $\mathrm{CA}$ negotiation and CA validation, see Figure 3. The number of bits for these parts were calculated and used as the basic "building blocks". This provided an estimate of transferred number of bits for the messages in the sequence of actions for attachment and negotiations for one user.

The first part of scalability analysis deals with the activity level of one user where a high degree of "greediness" implies that "many" service offers are evaluated and that the user always starts simultaneous multi-round CA negotiation with several providers. The load for a "nice user" is compared to that for a "greedy" user.

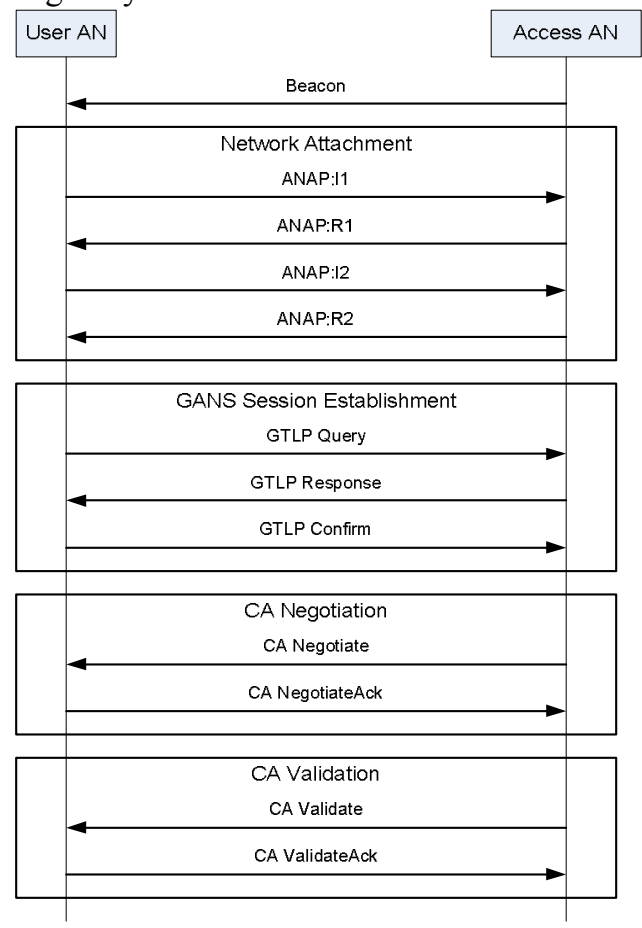

Figure 3: Signaling Sequence 
The second part of analysis looks at load as a function of both the number of users and the number of providers. The absolute numbers for the estimated signaling load are compared to "usage load" for data sessions in order to obtain relative load estimates.

\section{LOAD AND DELAy ANALYSIS RESUlTS}

The cost associated with network composition has been evaluated for one specific scenario using a combination of analytical calculations and simulations. The scenario used for this purpose is shown in Figure 4. It consists of one or more network operators (access ANs) offering Internet connectivity to one or more end users (user ANs).

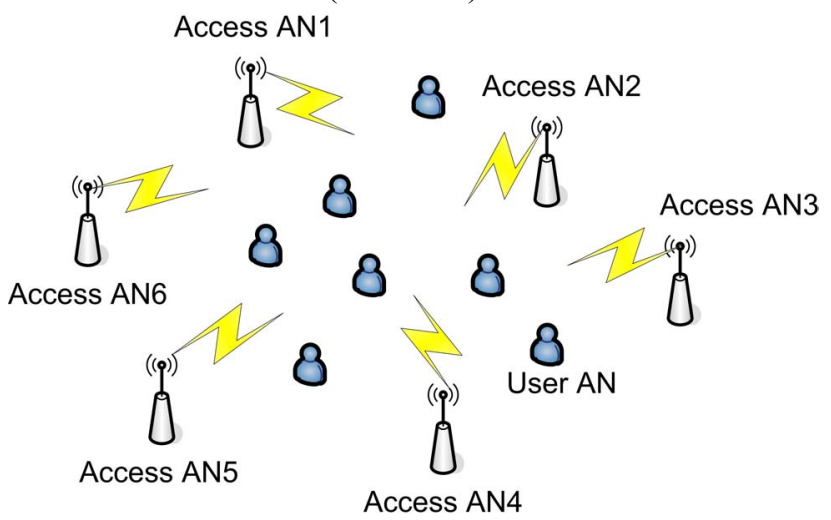

Figure 4: Simulation Scenario

We considered two types of costs for this analysis: signalling load and additional delay introduced on account of composition. For the first, the load was split into two components: "fixed" costs due to network attachment, CA validation and realization and "variable" costs arising from CA negotiations with one or more access networks involving multiple round-trips. Furthermore, load was computed for different user activity levels and "willingness to negotiate" (WTN). The former is expressed in terms of no. of access attempts per unit time while the latter represents different negotiation strategies. In particular, a high WTN means that end users negotiate "more" with several available networks whereas a low WTN implies negotiating "less" with only a few networks.

Figure 5 shows the load arising from negotiation and the fixed component (marked "Other signalling") for different use cases characterised by the parameters "M" and "q", where $\mathrm{M}$ equals the number of access networks the user $\mathrm{AN}$ is negotiating with and $\mathrm{q}$ is the number of rounds per negotiation. Note that the Mq product is fixed for a fair comparison (except for $\mathrm{M}=1, \mathrm{q}=1$ case which is the reference scenario). We can see from the graph of Figure 5 that negotiating with many networks consumes more resources than the strategy of negotiating more with fewer networks. The explanation is that the attachment and other "first time" signaling require more data than multiple negotiation rounds. Figure 6 shows the relative signalling load with respect to user data exchanged after composition in the course of a 128 kbit/s Constant Bit Rate session between an end user and a correspondent node (not shown in Figure 4). Note that the load due to composition-signalling is very small even for high values of $M(q=2$ here), see Figure 5. Furthermore, we assume only one application session whereas in general, end users will have several of these during the lifetime of a composition.

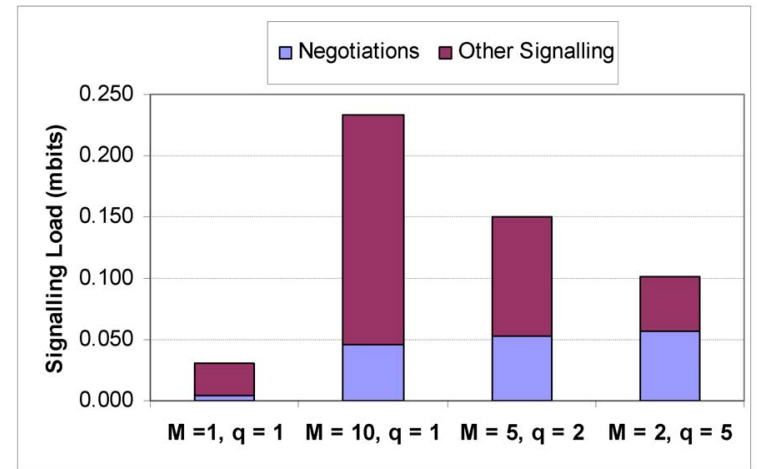

Figure 5: Signalling load

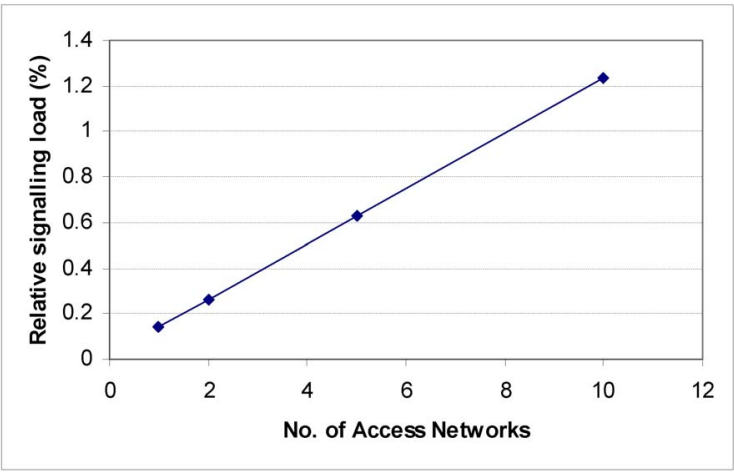

Figure 6: Relative signalling load

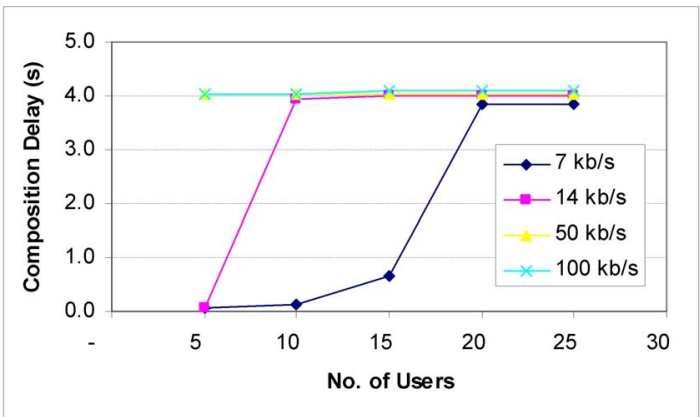

Figure 7: Composition-related latency

Finally, the latency introduced by the composition process is shown in Figure 7. A simplified model of AN was simulated, comprising the control functions necessary for carrying out composition procedures. The simulation scenario was similar to the one shown in Figure 4 except that only one access AN was present and all users were composing with it. The delay is measured for one particular user trying to compose with a specific access AN. The remaining users are assumed to have already composed with it and are participating in CBR sessions with correspondent hosts (data rates indicated in the graph). The delay experienced by the "new" user increases with background traffic and hits a saturation level which is independent of the total number of users and the background traffic rate. We conclude that delay is of particular concern, especially considering that such compositions will mainly happen in mobile networks where minimising handover delay is crucial. 


\section{CONCLuSions}

In future mobile communications networks using Ambient Networks (AN) technology, users will be able to connect to a multitude of networks operated by different business entities. The enabling technology includes functionality for advertising of access and service offers, multi-radio access support and flexible procedures for network selection, roaming and negotiation of terms and conditions for attachment to networks. Ambient Networks provides a tool called network composition that allows heterogeneous networks to cooperate automatically. This co-operation includes both business and technical aspects and each relation is described by a Composition Agreement between the networks or business entities

A major research challenge in the ongoing development of Ambient Networks is to ensure that the new functionality will not be "too complex" and introduce "too large" transactions costs for the end-user. In this paper, we considered scenarios where many users receive and evaluate "many" offers from a multitude of providers and also initiate "many" negotiations. In order to evaluate the benefits and feasibility of $\mathrm{AN}$, we have investigated the additional complexity in terms of signalling load and delay as experienced by the end user.

The results show that the most important parameters are the number of attachment attempts per time unit. The contribution to the signaling load from CA negotiations is less than for the attachment procedure. Furthermore, the overall load is a very small fraction of the total traffic exchanged between the end users and the service providers. The relative load of business related signaling is less then $1.0 \%$ even for the worst case of the investigated scenarios.

The delay introduced by the composition procedures is heavily dependent on the level of system load of the network under study. For low levels of background traffic, the delay is very small but increase in the number of users and/or data rates leads to a sharp jump, finally leading to a saturation level for the delay before the user sessions can start.

There are two key conclusions from the analysis presented here. First, the signalling load due to composition is very small, especially when considered in relation to application data exchanged afterwards, making the AN composition mechanisms a viable tool for dynamic network co-operation. Second, the delay introduced by composition is significantly large in some cases which indicates that composition may be useful in many cases but should not be used in some others. Note that these conclusions are only valid for scenarios involving WLAN systems and one could argue that the delay arises due to the inherent bandwidth bottlenecks of the wireless access system. Nevertheless, there is still much scope in the further optimisation of the composition process and there are indications that the delay can be much further reduced.

The work reported here is part of an ongoing comprehensive study of the costs associated with the introduction of Ambient Network functionality. The focus here was mainly on CA negotiation. Further work will consider the effect of advertising and discovery process. The impact of different advertising strategies on signalling load will be studied. In addition, the scalability analysis will be extended to cover scenarios with other radio access technologies, especially UMTS systems and hybrid systems such as WLAN-UMTS.

\section{ACKNOWLEDGMENTS}

The authors would like to thank their colleagues in the Ambient Networks project, especially the partners in the Performance Evaluation group.

\section{REFERENCES}

[1] http://www.fon.com

[2] http://www.ambient-networks.org

[3] Ambient Networks System Description, Public Deliverable D-A.2, January 2007, available online from www.ambient-networks.org

[4] Connecting Ambient Networks - Architecture and Protocol Design, Public Deliverable D-3.2, March 2005, available online from http://www.ambient-networks.org

[5] Design of Composition Framework, Public Deliverable D-G.1, November 2006, available online from www.ambient-networks.org

[6] Teemu Rinta-aho, R. Campos, A. Mehes, U. Meyer, J. Sachs, and G. Selander, Ambient Network Attachment, in Proceedings of the 16th IST Mobile and Wireless Communications Summit, July 2007.

[7] Johnson P, "A Glossary of Political Economy"; online edition at http://www.auburn.edu/ johnspm/gloss/, Auburn University. 